\title{
Mortality after Staphylococcus aureus bacteraemia in two hospitals in Oxfordshire, 1997-2003: cohort study
}

\author{
David H Wyllie, Derrick W Crook, Tim E A Peto
}

\begin{abstract}
Objective To determine the incidence of methicillin resistant and methicillin sensitive Staphylococcus aureus (MRSA and MSSA) bacteraemia in inpatients and associated mortality within 30 days after diagnosis.

Design Anonymised record linkage study of data from hospital information systems and microbiology databases.

Setting Teaching hospital and district general hospital in Oxfordshire.

Participants Inpatients aged 18 or over admitted to a teaching hospital between 1 April 1997 and 31 March 2004 and to a district general hospital between 1 April 1999 and 31 March 2004. The main part of the study comprised 216644 inpatients; patients admitted to haematology, nephrology, or oncology services were not included because most were managed as outpatients.

Outcome measures Nosocomial MSSA and MRSA

bacteraemia; death in hospital within 30 days after bacteraemia. Results Rates of $S$ aureus bacteraemia rose between 1997 and 2003, and MRSA was responsible for this increase. Overall mortality 30 days after bacteraemia was $29 \%$. The crude odds ratio for death after MRSA bacteraemia compared with MSSA bacteraemia was 1.49 (95\% confidence interval 0.99 to 2.26). Conclusion The spread of MRSA has greatly increased the overall number of cases of $S$ aureus bacteraemia and has contributed to short term mortality after $S$ aureus bacteraemia.
\end{abstract}

\section{Introduction}

Rapidly rising rates of infection with methicillin resistant Staphylococcus aureus (MRSA) led to the revision of United Kingdom national infection control guidelines in 1998. Previously, an MRSA search and destroy method was used; afterwards, patients were stratified according to risk, and targeted prevention measures recommended. ${ }^{1}$ Rates of MRSA bacteraemia have continued to rise, however, causing governmental and professional concern. ${ }^{23}$

Our understanding of the impact of the high rate of MRSA in the UK on death after $S$ aureus bacteraemia is limited. Analyses of death certificates have suggested rising MRSA associated mortality; however, death certificate analysis can result in biased estimates of disease associated mortality. ${ }^{4}$ Here, we describe secular trends in nosocomial methicillin sensitive $S$ aureus (MSSA) bacteraemia, MRSA bacteraemia, and survival 30 days after bacteraemia in inpatients in acute care hospitals in Oxfordshire after the search and destroy policy changed, and we discuss the impact of the MRSA epidemic on mortality after nosocomial $S$ aureus bacteraemia.

\section{Methods}

Data sources, linkage, and statistical analysis

Our study took place in the John Radcliffe Hospital, the Radcliffe Infirmary, and the Churchill Hospital, which form one teaching hospital in Oxford and offer all specialist regional services, such as cardiothoracic surgery, neurosurgery, and nephrology. We also studied the Horton Hospital, a district general hospital in Banbury, $48 \mathrm{~km}$ north of Oxford. Together, they provide acute clinical and bacteriology services to about 600000 people. Other hospitals in the area include a specialist orthopaedic hospital, psychiatric hospitals, and several community hospitals. Admissions to these other hospitals were not included in our study.

Our approach relied on anonymised record linkage. ${ }^{6}$ Briefly, we generated a database of all patient admissions, excluding outpatients, in the Oxford hospitals between 1 January 1997 and 31 March 2004 and in the Horton Hospital between 1 January 1999 and 31 March 2004. We chose two different time periods because the two sites used different administration systems before 1999 . In addition to admission data, the database had information on all isolates of MSSA and MRSA detected between 1 January 1995 and 31 March 2004.

\section{Cohort studied}

The patients analysed were admitted between 1 April 1997 and 31 March 2004 to the Oxford hospitals or between 1 April 1999 and 31 March 2004 to the Horton Hospital; they came from the community (they had not been transferred from another hospital); and they were aged 18 or over on the day of admission. We made this age restriction because we wanted to use mortality as an outcome measure, and preliminary analysis showed that mortality was too low in people under 18 to be a reliable marker of the effect of $S$ aureus bacteraemia (data not shown).

We restricted our main analysis to patients who stayed in hospital for two or more days, as these patients are at risk of nosocomial bacteraemia. ${ }^{7}$ We did not analyse patients who were treated by the haematology, oncology, or nephrology departments because they are often treated as day cases rather than as inpatients. ${ }^{67}$

\section{Screening policies and microbiology}

Our MRSA screening policies were based on national guidance; MRSA screening at admission was recommended in high risk units (renal services, intensive care, and some surgical wards). ${ }^{1}$ Microbiological processing used standard techniques, as recommended by the standard operating procedures of the Health Protection Agency. ${ }^{6}$

Statistical analysis

We plotted secular trends in dichotomous variables ( $S$ aureus bacteraemia, inpatient death, previous isolation of MRSA) and 
Table 1 Cases of Staphylococcus aureus bacteraemia in two Oxfordshire hospitals, 1997-2003

\begin{tabular}{|c|c|c|c|c|}
\hline \multirow[b]{2}{*}{ Bacteraemia } & \multicolumn{2}{|c|}{ Patients admitted to renal, haematology, and oncology services } & \multicolumn{2}{|c|}{ Patients admitted to other departments } \\
\hline & $\begin{array}{l}\text { All patients admitted* } \\
(\mathrm{n}=221036)\end{array}$ & $\begin{array}{l}\text { Patients who stayed } \geq 2 \text { days }(\mathrm{n}=16 \\
322)\end{array}$ & $\begin{array}{l}\text { All patients admitted }{ }^{*}(\mathrm{n}=493 \\
500)\end{array}$ & $\begin{array}{c}\text { Patients who stayed } \geq 2 \text { days } \quad \text { ( } \mathrm{n}=216 \\
644 \text { ) }\end{array}$ \\
\hline No & 220851 & 16254 & 493155 & 216183 \\
\hline MRSA & 31 & 19 & 78 & 232 \\
\hline MSSA & 154 & 49 & 267 & 229 \\
\hline
\end{tabular}

MRSA=methicillin resistant $S$ aureus, MSSA=methicillin sensitive $S$ aureus.

* Patients who had MRSA or MSSA were diagnosed on day 0 or 1 of admission.

†Patients who had MRSA or MSSA had nosocomial disease (occurred $\geq 2$ days after admission).

continuous variables (mean age, duration of stay) in blocks of six months, and we stratified the data by specialty. We used $\chi^{2}$ tests to analyse relations between $S$ aureus bacteraemia, inpatient death, and previous isolation of MRSA. To examine changes in continuous variables (age, duration of stay) over time, we used linear regression to relate the variable of interest to date of admission (represented as a decimal), with date as the sole explanatory variable. For $S$ aureus bacteraemia, we used a similar approach with logistic regression.

We also built two sets of multivariate logistic regression models; one described $S$ aureus bacteraemia, and the other described inpatient death according to patient characteristics. Variables that were significant on univariate analysis were added to the multivariate models. We plotted stratified observations and model predictions to check that the variables studied were adequately modelled in linear terms. Modelling with duration of inpatient stay was performed after log transformation, as this resulted in improved fit. We tested the contribution of interaction terms by adding each term individually to a multivariate model without interaction terms. Contributing interactions were then added to a final multivariate model. The results are shown as odds ratio ( $95 \%$ confidence interval).

We used a $\chi^{2}$ test to compare inpatient mortality 30 days after bacteraemia among patients with MSSA and MRSA. We stratified by several variables and used Breslow-Day tests to look for evidence of heterogeneity of mortality with MRSA relative to MSSA between strata. SPSS 12.0 for Windows was used for all analyses.

\section{Definitions}

- Case of $S$ aureus bacteraemia-isolation of $S$ aureus from blood cultures or isolation of this organism within the two previous weeks (as recommended by the UK mandatory surveillance scheme $)^{2}$

- Nosocomial $S$ aureus bacteraemia-a case of $S$ aureus bacteraemia arising two or more days after admission (as recommended by the Centers for Disease Control $)^{7}$

- Previous isolation of MRSA-MRSA isolated from any sample at any time between 1 January 1995 and the date of admission

- Intensive care unit: general adult intensive care unit, neurology intensive care unit, and cardiothoracic recovery unit. Coronary care units were not considered to be intensive care units. Our hospitals do not have separate adult general high dependency units

- Death during admission-death of a patient during the current episode of hospital care. An episode starts with admission and ends when the patient is discharged

- Inpatient mortality within 30 days of bacteraemia-death of an inpatient within 30 days of meeting the criteria for being a case of $S$ aureus bacteraemia. We chose a follow-up of 30 days since this period is a time of high risk after bacteraemia. ${ }^{8}$ The event was recorded whether or not the patient had been discharged between bacteraemia and death

\section{Results}

Table 1 shows the number of cases of $S$ aureus bacteraemia during our study. Patients treated by renal, haematology, and oncology services were at high risk of $S$ aureus bacteraemia-they accounted for 253 of the 1059 cases (24\%). Because only $7 \%$ (16322/221 036) of patients admitted to renal, haematology, and oncology services stayed for two or more days (table 1), these patients were not thought to be appropriate for use in our study of nosocomial bacteraemia (see box for definition). ${ }^{6}$ We excluded this group from the rest of our analysis.

After excluding these patients, our study cohort was made up of 216644 admissions. We identified 461 cases of nosocomial $S$ aureus bacteraemia-232 cases of MRSA and 229 cases of MSSA. Of these 461 cases, 441 (96\%) occurred in the 144134 admissions to medical, surgical, or trauma services (fig 1). Only 20 of 461 cases $(4 \%)$ occurred in the other 72510 patients. We cannot make robust estimates of bacteraemia rates among these 72510 patients, who form a heterogeneous group although most (54 031) patients were admitted to obstetrics or gynaecology. We restricted our further analyses to medical, surgical, and trauma specialties.

\section{Rates of MRSA and MSSA bacteraemia}

During the seven years from 1997, overall rates of $S$ aureus bacteraemia during each admission rose; this was due to an
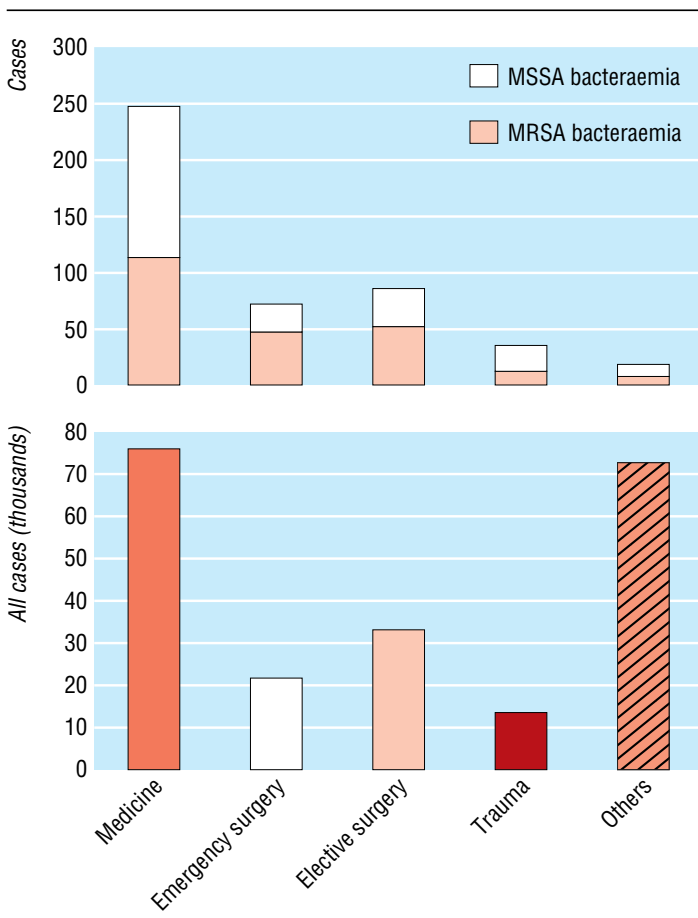

Fig 1 Admissions and cases of bacteraemia in two Oxfordshire hospitals, 1997-2003 


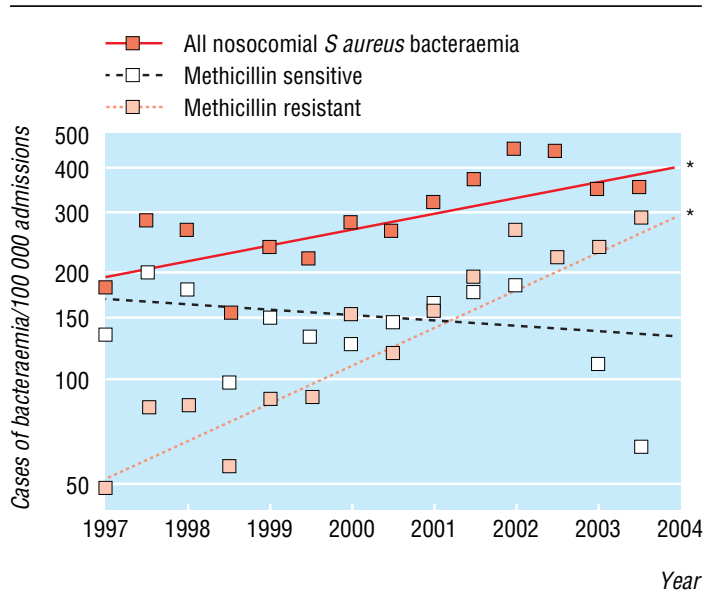

Fig 2 Changes in rates of nosocomial bacteraemia over time in two Oxfordshire hospitals ( $n=144134)$. Regression lines indicate change over time. Asterisks indicate that the slope of the line is significant $(P<0.01 ; P>0.20$ for the others)

increase in MRSA bacteraemia-rates of MSSA bacteraemia did not alter (fig 2). Other changes also occurred during this time. The number of patients admitted with a history of MRSA increased (fig 3), and these patients had higher rates of nosocomial bacteraemia with MRSA but not MSSA (fig 4). The age of patients increased (fig 3); age was strongly associated with bacteraemia rates (fig 5). The duration of inpatient stay, which is also associated with rates of bacteraemia per admission, increased (figs 3 and 5).

\section{Increase in $S$ aureus bacteraemia and isolation of MRSA}

We used logistic regression to investigate how increased rates of bacteraemia might be explained by changes in case mix. Variables considered included specialty-which was not associated with rates of bacteraemia (table 2)-and age, previous isolation of MRSA, and duration of stay-which were associated with rates of bacteraemia. Table 2 shows a multivariate model for all types of $S$ aureus bacteraemia; we constructed similar models for MSSA and MRSA bacteraemia (not shown). These models show that the increase in overall $S$ aureus bacteraemia is not explained by changes in case mix alone (adjusted increase each year 1.06, 1.01 to $1.12 ; \mathrm{P}=0.02$; table 2 ). The estimated rates of change per annum for MRSA and MSSA bacteraemia were 1.22 (1.13 to $1.31)$ and $0.93(0.87$ to $1.00 ; \mathrm{P}=0.05)$. Thus, the increase in overall rates of $S$ aureus bacteraemia is due to MRSA; although unadjusted rates of MSSA bacteraemia have stayed constant over time, when correlates of increased risk, ageing hospital population, and increasing stay are adjusted for, the rate of MSSA bacteraemia may have fallen.

The model in table 2 also shows a significant interaction between age and duration of stay, indicating that the increased risk associated with a longer period of inpatient stay depends on age and previous length of stay. We built more complex models that included specialty and examined multiple interactions. Results were similar to those presented in table 2.

\section{Mortality associated with $S$ aureus bacteraemia}

Next we asked whether MSSA and MRSA bacteraemia identified a group at increased risk of inpatient death. Figure 6 shows the observed risk of all cause mortality during admission, stratified by age and the year of admission. The increased risk associated with $S$ aureus bacteraemia persisted in a multivariate analysis that included age, date of admission, previous isolation of MRSA, and specialty (table 3).
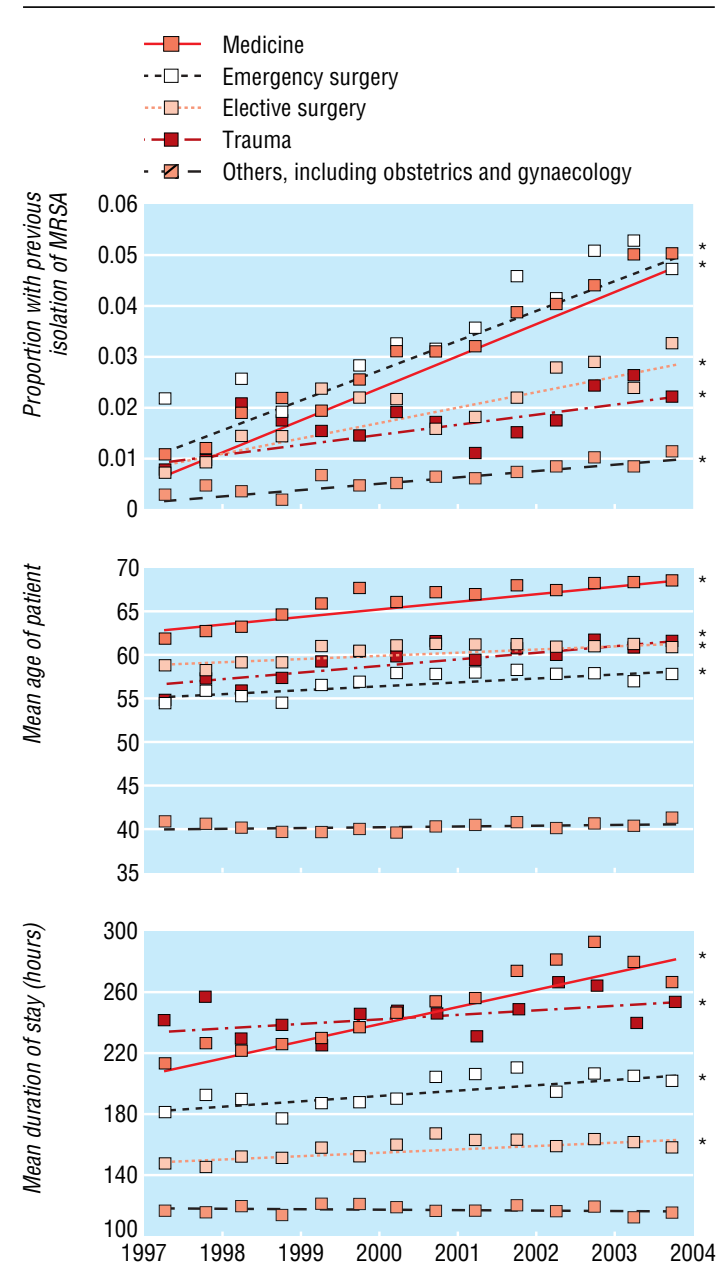

Year

Fig 3 Changes in previous isolation of methicillin resistant Staphyloccocus aureus (MRSA), age, and duration of stay over time in two Oxfordshire hospitals $(n=144134)$. Regression lines indicate change over time. Asterisks indicate that the slope of the line is significant $(P<0.01 ; P>0.20$ for the others)

We investigated the association between MRSA and MSSA bacteraemia and death during the next 30 days. Among the 441

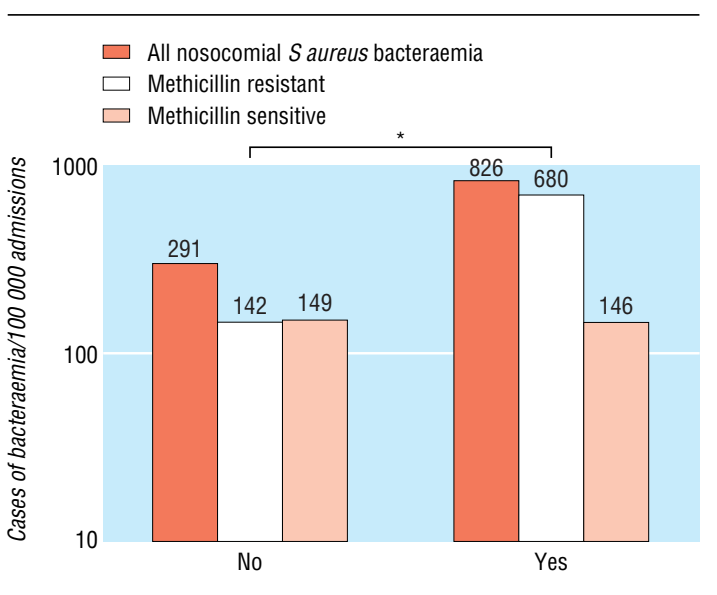

MRSA isolated before admission

Fig 4 Rates of nosocomial bacteraemia according to previous isolation of methicillin resistant Staphyloccocus aureus (MRSA) in two Oxfordshire hospitals $(n=144$ 134). $P<0.01$ 

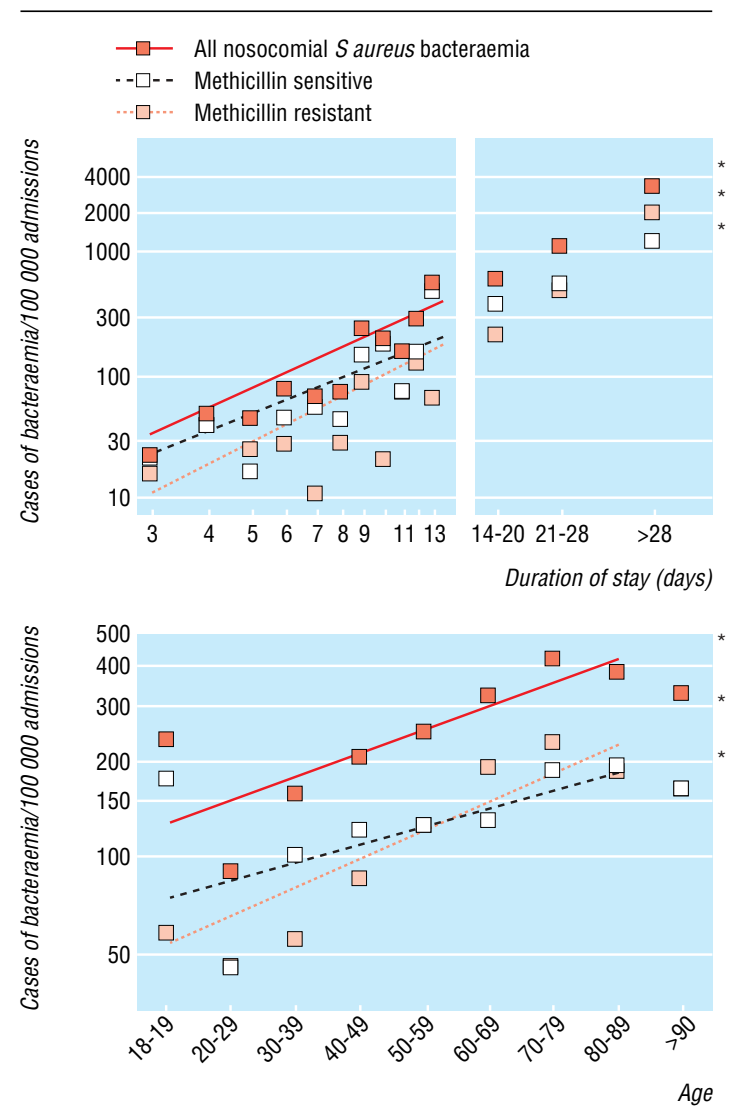

Fig 5 Rates of nosocomial bacteraemia according to duration of stay (top) and age (bottom) in two Oxfordshire hospitals ( $n=144134)$, log scale. Asterisks indicate that the slope of the regression line is significant $(P<0.01 ; P>0.20$ for the others) cases of $S$ aureus bacteraemia, 130 (29\%) patients died within 30 days. The death rate was 34\% (76/227) for MRSA and 27\% (58/ 214) for MSSA. The crude odds ratio for mortality within 30 days with isolation of MRSA versus MSSA was 1.49 (0.99 to 2.26). To identify groups in which MRSA was more likely to cause death than MSSA, we stratified by age, isolation of MRSA before admission, stay on an intensive care unit or renal ward before bacteraemia, specialty admitted to, specialty from which last discharged, and year of admission (fig 7). We found no significant heterogeneity within the strata examined; thus, the risk of death after MRSA bacteraemia is similar to (or higher than) that after MSSA bacteraemia in many patient groups.

\section{Discussion}

Similar to previous studies, we found that about $30 \%$ of patients in our study with $S$ aureus bacteraemia died within 30 days after diagnosis. ${ }^{9-11}$ From 1997 to 2003, instead of displacing MSSA as the cause of nosocomial $S$ aureus bacteraemia, MRSA added to it, thereby increasing the rates of $S$ aureus bacteraemia. This increase cannot be explained by ageing of the hospitalised population or increased hospital stay in the group studied, although both occurred. Our findings are reminiscent of observations made early in the MRSA epidemic, when increases in total hospital associated infection caused by MRSA occurred without changes in nosocomial infection caused by MSSA. ${ }^{12}$

Comparison of short term outcome (mortality within 30 days) after MRSA and MSSA bacteraemia suggests that the outcome of MRSA bacteraemia was at least as bad as that from MSSA bacteraemia. There may be an MRSA associated excess of the order of $50 \%$, although this excess is not conventionally significant in our study. A small MRSA associated excess in mortality would fit in with the findings of a UK based study of a larger

Table 2 Risk of nosocomial Staphylococcus aureus bacteraemia (both MRSA and MSSA) in two 0xfordshire hospitals 1997-2003 (n=144 134)

\begin{tabular}{|c|c|c|c|c|c|c|}
\hline \multirow[b]{2}{*}{ Variable } & \multicolumn{4}{|c|}{ Univariate analysis } & \multicolumn{2}{|c|}{ Multivariate analysis } \\
\hline & Odds ratio $(95 \% \mathrm{Cl})$ & Wald $\chi^{2}$ & df & $P$ value & Odds ratio $(95 \% \mathrm{Cl})$ & Wald $\chi^{2}(1 \mathrm{df})$ \\
\hline Secular trend, per year & 1.11 (1.05 to 1.17$)$ & 18.2 & 1 & $<0.01$ & 1.06 (1.01 to 1.12) & 5.1 \\
\hline Specialty, overall & - & 3.6 & 3 & 0.30 & Not entered & - \\
\hline Log (length of stay in weeks) & 4.33 (3.99 to 4.70$)$ & 1251 & 1 & $<0.01$ & 11.3 (8.10 to 15.6) & 210 \\
\hline Age, per decade & 1.19 (1.12 to 1.25$)$ & 38.8 & 1 & $<0.01$ & 1.09 (1.01 to 1.18$)$ & 4.3 \\
\hline Previous isolation of MRSA & 2.86 (2.01 to 4.06) & 34.2 & 1 & $<0.01$ & 1.53 (1.07 to 2.21) & 5.4 \\
\hline Log (duration of stay) × age & 1.18 (1.17 to 1.20$)$ & 1169 & 1 & $<0.01$ & 0.87 (0.83 to 0.91$)$ & 34 \\
\hline
\end{tabular}

MRSA=methicillin resistant $S$ aureus, MSSA=methicillin sensitive $S$ aureus.
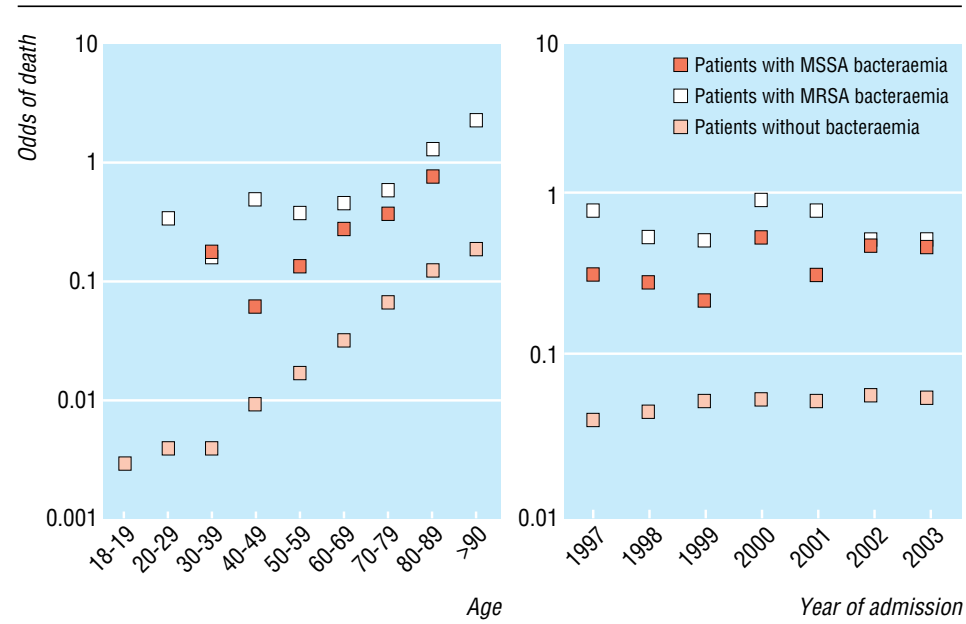

Fig 6 Risk of death for inpatients in two Oxfordshire hospitals ( $n=144134$ ) according to age and year 
Research

Table 3 Risk of death during inpatient stay in 144134 patients in two Oxfordshire hospitals 1997-2003

\begin{tabular}{|c|c|c|c|c|c|c|}
\hline \multirow[b]{2}{*}{ Variable } & \multicolumn{3}{|c|}{ Univariate analysis } & \multicolumn{3}{|c|}{ Multivariate analysis } \\
\hline & Odds ratio $(95 \% \mathrm{Cl})$ & Wald $\chi^{2}$ & df & Odds ratio $(95 \% \mathrm{CI})$ & Wald $\chi^{2}$ & df \\
\hline Secular trend (per year) & 1.06 (1.04 to 1.07$)$ & 86 & 1 & 1.01 (1.00 to 1.02$)$ & 2.2 & 1 \\
\hline Overall nosocomial $S$ aureus bacteraemia & - & 506 & 2 & - & 419 & 2 \\
\hline Nosocomial MRSA bacteraemia & $12.30(9.42$ to 16.10$)$ & 339 & 1 & 13.80 (10.20 to 18.60$)$ & 288 & 1 \\
\hline Nosocomial MSSA bacteraemia & 7.30 (5.40 to 9.84$)$ & 169 & 1 & 7.04 (5.05 to 9.80$)$ & 133 & 1 \\
\hline Age (per decade) & 1.92 (1.88 to 1.95$)$ & 4315 & 1 & 1.79 (1.75 to 1.82$)$ & 3473 & 1 \\
\hline Previous isolation of MRSA & 1.95 (1.75 to 2.17) & 149 & & 1.39 (1.24 to 1.56$)$ & 33 & 1 \\
\hline Specialty overall (relative to medicine) & - & 1798 & 3 & - & 1065 & 3 \\
\hline Trauma & $0.41(0.37$ to 0.45$)$ & 310 & 1 & 0.473 (0.43 to 0.52$)$ & 210 & 1 \\
\hline Elective surgery & $0.12(0.10$ to 0.13$)$ & 1353 & 1 & 0.170 (0.15 to 0.19$)$ & 887 & 1 \\
\hline Emergency surgery & 0.49 (0.46 to 0.53$)$ & 359 & 1 & 0.729 (0.68 to 0.79$)$ & 67 & 1 \\
\hline
\end{tabular}

MRSA=methicillin resistant $S$ aureus, MSSA=methicillin sensitive $S$ aureus.

number of risk factors and with a recent meta-analysis. ${ }^{13}{ }^{14}$ Thus the emergence of MRSA seems to be associated with an increase in inpatient mortality soon after $S$ aureus bacteraemia.

\section{Limitations}

We did not consider long term outcomes, such as readmission or relapse, and this would be an important area for future study. We

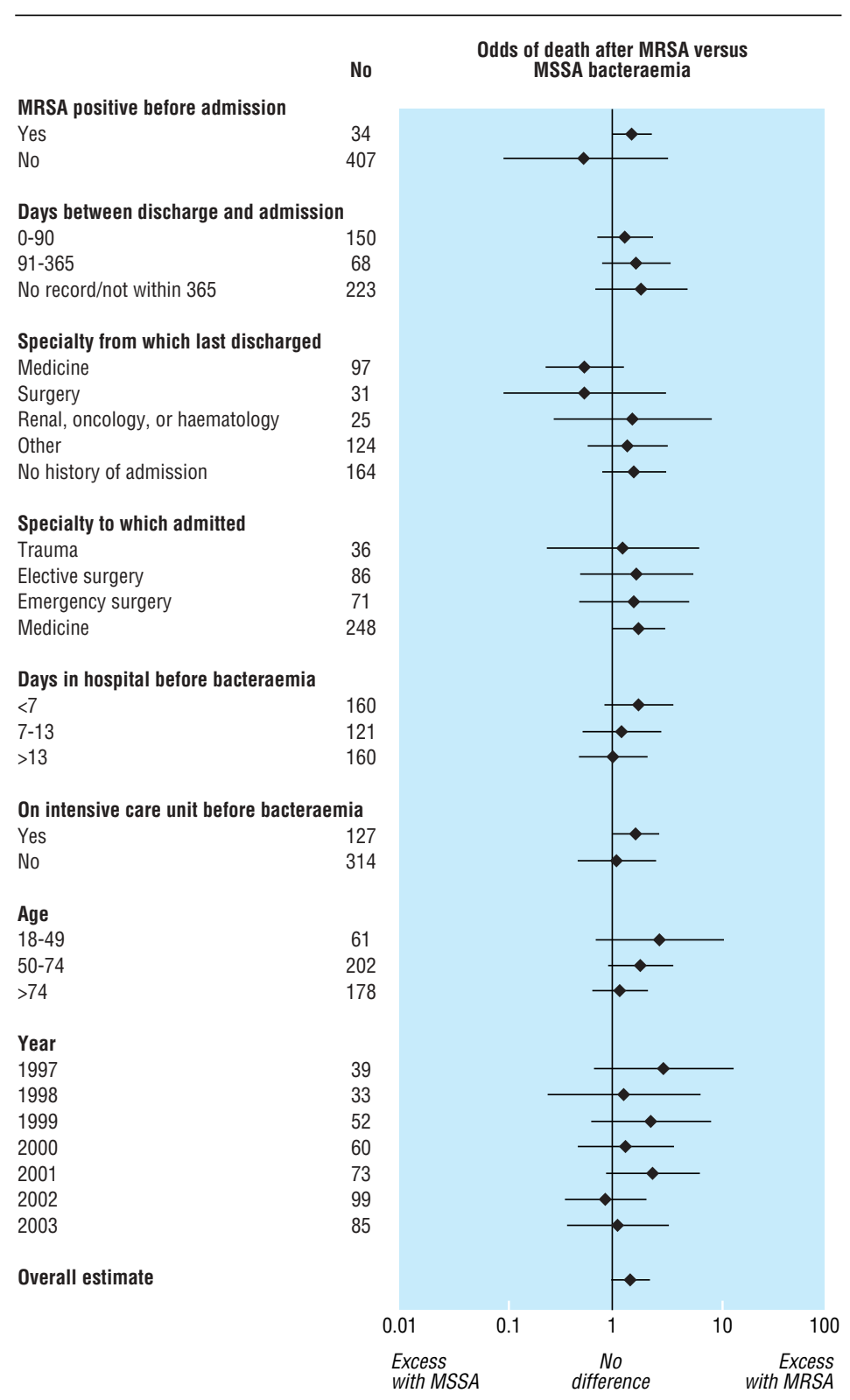

Fig 7 Risk of death 30 days after diagnosis of Staphylococcus aureus bacteraemia in two 0xfordshire hospitals ( $n=144$ 134) 


\section{What is known about this topic}

Mortality is high in inpatients who develop methicillin resistant Staphylococcus aureus (MRSA) bacteraemia-short term mortality is $12-25 \%$

\section{What this study adds}

From 1997 to 2003, hospital acquired S aureus bacteraemia rates and post-bacteraemia mortality rose because of an increase in the number of MRSA cases

Mortality after MRSA bacteraemia was at least as high as after MSSA bacteraemia

lack information on antibiotic treatment, which is a determinant of outcome in bloodstream infection in general and $S$ aureus in particular. ${ }^{10}{ }^{15}{ }^{16}$ Within our institution, the combined infectious diseases and microbiology service reviews and advises on a common treatment strategy for MRSA bacteraemia, as advised by the Infectious Diseases Society of America. ${ }^{17}$ This comprises drainage of any sources, removal of intravascular catheters, and intravenous glycopeptides for two to four weeks (with the length of time depending on the presence or absence of a removable source of infection). Little variation occurred in treatment, so we cannot examine the effect of such variations. However, in a larger multicentre surveillance programme investigating mortality after $S$ aureus bacteraemia, treatment would be an important issue to examine.

A key issue is whether bacteraemia was the underlying cause of death in these patients. It is difficult to attribute the cause of death in cases of $S$ aureus bacteraemia because of the many coexisting risk factors for death, such as acute and chronic illness, and the high degree of medical intervention. ${ }^{18}$ Because we have limited information on comorbidity, we did not try to assess the fraction of deaths attributable to $S$ aureus bacteraemia. The proportion of deaths that can be attributed to MRSA bacteraemia has been investigated in several settings and is substantial. Multivariate methods yielded estimates of $25 \%$ in a Belgian intensive care unit and $15 \%$ in older patients in a study from the United States. ${ }^{9}{ }^{19}$ A UK study using clinical judgment to attribute the cause of death produced an estimate of $12 \%{ }^{13}$

\section{Implications}

The contribution of MRSA to overall $S$ aureus bacteraemia combined with the high mortality associated with the condition supports current attempts to reduce the spread of MRSA. It would be useful to carry out studies with a similar design to ours that systematically collect hospital based, patient centred data on cases of MSSA and MRSA and controls. Such an approach would be analogous to the surveillance operating after high risk cardiac and other surgery, and it would allow accurate, timely feedback to clinicians and patients of current practice and disease burden..$^{20}$ If implemented over a sufficient patient base, it would allow validation of observations on the excess mortality associated with MRSA, investigation of reasons for MRSA associated excess mortality, estimation of the effect of treatment protocols, and ecological and individual studies of determinants of outcome. ${ }^{13}{ }^{14}$ It would also allow planning of adequately powered cluster randomised intervention trials of the options available to prevent the spread of MRSA and treat bacteraemia.
We thank the reviewers for their helpful comments.

Contributors: DHW obtained and linked the data and wrote the paper. DHW and TEAP analysed the data. All authors contributed to the paper. DHW is guarantor.

Funding: None.

Competing interests: None declared.

Ethical approval: Not needed.

1 British Society for Antimicrobial Chemotherapy, Hospital Infection Society and the Infection Control Nurses Association. Revised guidelines for the control of methicillinresistant Staphylococcus aureus infection in hospitals.J Hosp Infect 1998;39:253-90.

2 Department of Health. MRSA surveillance system: results. www.dh.gov.uk/ PublicationsAndStatistics/Publications/PublicationsStatistics/ PublicationsStatisticsArticle/fs/en?CONTENT_ID $=4085951 \&$ chk $=$ HBt2QD (accessed 20 May 2006).

3 Reid J. Bloodborne MRSA infection rates to be halved by 2008. www.dh.gov.uk PublicationsAndStatistics/PressReleases/PressReleasesNotices/fs/ en?CONTENT ID = 4093533\&chk $=$ MY\% $\%$ BkD $/($ accessed 27 Apr 2006)

4 Griffiths C, Lamagni TL, Crowcroft NS, Duckworth G, Rooney C. Trends in MRSA in England and Wales: analysis of morbidity and mortality data for 1993-2002. Health Stat Q 2004(21):15-22.

5 Crowcroft NS, Catchpole M. Mortality from methicillin resistant Staphylococcus aureus in England and Wales: analysis of death certificates. BMJ 2002;325:1390-1.

6 Wyllie DH, Peto TE, Crook D. MRSA bacteraemia in patients on arrival in hospital: a cohort study in Oxfordshire 1997-2003. BMJ 2005;331:992-5.

7 Garner JS, Jarvis WR, Emori TG, Horan TC, Hughes JM. CDC definitions for nosocomial infections, 1988. Am J Infect Control 1988;16:128-40.

8 Bates DW, Pruess KE, Lee TH. How bad are bacteremia and sepsis? Outcomes in a cohort with suspected bacteremia. Arch Intern Med 1995; 155:593-8.

9 McClelland RS, Fowler VG Jr, Sanders LL, Gottlieb G, Kong LK, Sexton DJ, et al. Staphylococcus aureus bacteremia among elderly vs younger adult patients: comparison phylococcus aureus bacteremia among elderly vs younger adult pate
of clinical features and mortality. Arch Intern Med 1999:159:1244-7.

10 Jensen AG, Wachmann CH, Espersen F, Scheibel J, Skinhoj P, Frimodt-Moller N. Treatment and outcome of Staphylococcus aureus bacteremia: a prospective study of 278 cases. Arch Intern Med 2002;162:25-32.

11 Fatkenheuer G, Preuss M, Salzberger B, Schmeisser N, Cornely OA, Wisplinghoff H, et al. Long-term outcome and quality of care of patients with Staphylococcus aureus bacteremia. Eur J Clin Microbiol Infect Dis 2004;23:157-62.

12 Stamm AM, Long MN, Belcher B. Higher overall nosocomial infection rate because of increased attack rate of methicillin-resistant Staphylococcus aureus. Am J Infect Control $1993 ; 21: 70-4$

13 Melzer M, Eykyn SJ, Gransden WR, Chinn S. Is methicillin-resistant Staphylococcus aureus more virulent than methicillin-susceptible $\mathrm{S}$. aureus? A comparative cohort study of British patients with nosocomial infection and bacteremia. Clin Infect Dis study of British p
2003;37:1453-60.

14 Cosgrove SE, Sakoulas G, Perencevich EN, Schwaber MJ, Karchmer AW, Carmeli Y. Comparison of mortality associated with methicillin-resistant and methicillinsusceptible Staphylococcus aureus bacteremia: a meta-analysis. Clin Infect Dis 2003;36:53-9.

15 Hanon FX, Monnet DL, Sorensen TL, Molbak K, Pedersen G, Schonheyder H. Survival of patients with bacteraemia in relation to initial empirical antimicrobial treatment Scand J Infect Dis 2002;34:520-8.

16 Fowler VG Jr, Sanders LL, Sexton DJ, Kong L, Marr KA, Gopal AK, et al. Outcome of Staphylococcus aureus bacteremia according to compliance with recommendations of infectious diseases specialists: experience with 244 patients. Clin Infect Dis 1998;27: infectiou

17 Mermel LA, Farr BM, Sherertz RJ, Raad, II, O'Grady N, Harris JS, et al. Guidelines for the management of intravascular catheter-related infections. I Intraven Nurs the management

18 Noskin GA, Rubin RJ, Schentag JJ, Kluytmans J, Hedblom EC, Smulders M, et al. The burden of Staphylococcus aureus infections on hospitals in the United States: an analysis of the 2000 and 2001 nationwide inpatient sample database. Arch Intern Med 2005; 165:1756-61.

19 Blot SI, Vandewoude KH, Hoste EA, Colardyn FA. Outcome and attributable mortality in critically ill patients with bacteremia involving methicillin-susceptible and methicillin-resistant Staphylococcus aureus. Arch Intern Med 2002;162:2229-35.

20 Wilson AP, Gibbons C, Reeves BC, Hodgson B, Liu M, Plummer D, et al. Surgical wound infection as a performance indicator: agreement of common definitions of wound infection as a performance indicator: agreen
wound infection in 4773 patients. BMJ 2004;329:720.

21 Keogh B, Spiegelhalter D, Bailey A, Roxburgh J, Magee P, Hilton C. The legacy of Bristol: public disclosure of individual surgeons' results. BMJ 2004;329:450-4. (Accepted 13 April 2006)

doi $10.1136 /$ bmj.38834.421713.2F

Nuffield Department of Clinical Laboratory Sciences, University of Oxford, John Radcliffe Hospital, Oxford OX3 9DU

David H Wyllie clinical lecturer in microbiology

Derrick W Crook consultant microbiologist

Nuffield Department of Medicine, University of Oxford

Tim E A Peto professor of infectious diseases

Correspondence to: D Wyllie david.wyllie@ndcls.ox.ac.uk 